Article

\title{
Swiprosin-1/EFhD-2 Expression in Cardiac Remodeling and Post-Infarct Repair: Effect of Ischemic Conditioning
}

\author{
Zoltán Giricz ${ }^{1,2}$, András Makkos ${ }^{1}$, Rolf Schreckenberg ${ }^{3}$, Jochen Pöling ${ }^{4}$, Holger Lörchner ${ }^{4}$, \\ Krisztina Kiss ${ }^{5}$, Péter Bencsik ${ }^{2,5}{ }^{(0)}$, Thomas Braun ${ }^{4}\left(\mathbb{D}\right.$, Rainer Schulz ${ }^{3}$, Péter Ferdinandy ${ }^{1,2}$ \\ and Klaus-Dieter Schlüter ${ }^{3, *(D)}$ \\ 1 Cardiometabolic Research Group and MTA-SE System Pharmacology Research Group \\ Department of Pharmacology and Pharmacotherapy, Semmelweis University, 1085 Budapest, Hungary; \\ zoltan.giricz@pharmahungary.com (Z.G.); andras.makkos@pharmahungary.com (A.M.); \\ peter.ferdinandy@pharmahungary.com (P.F.) \\ 2 Pharmahungary Group, H-6720 Szeged, Hungary; peter.bencsik@pharmahungary.com \\ 3 Department of Physiology, Justus-Liebig-University, 35390 Giessen, Germany; \\ rolf.schreckenberg@physiologie.med.uni-giessen.de (R.S.); \\ rainer.schulz@physiologie.med.uni-giessen.de (R.S.) \\ 4 Department of Cardiac Development and Remodeling, Max Planck Institute for Heart and Lung Research, \\ Ludwigstrasse 43, 61231 Bad Nauheim, Germany; Jochen.Poeling@mpi-bn.mpg.de (J.P.); \\ Holger.Loerchner@mpi-bn.mpg.de (H.L.); Thomas.Braun@mpi-bn.mpg.de (T.B.) \\ 5 Department of Pharmacology and Pharmacotherapy, University of Szeged, H-6721 Szeged, Hungary; \\ krisztina.kiss@pharmahungary.com \\ * Correspondence: Klaus-Dieter.Schlueter@physiologie.med.uni-giessen.de
}

Received: 8 April 2020; Accepted: 4 May 2020; Published: 9 May 2020

\begin{abstract}
Swiprosin-1 (EFhD2) is a molecule that triggers structural adaptation of isolated adult rat cardiomyocytes to cell culture conditions by initiating a process known as cell spreading. This process mimics central aspects of cardiac remodeling, as it occurs subsequent to myocardial infarction. However, expression of swiprosin-1 in cardiac tissue and its regulation in vivo has not yet been addressed. The expression of swiprosin-1 was analyzed in mice, rat, and pig hearts undergoing myocardial infarction or ischemia/reperfusion with or without cardiac protection by ischemic preand postconditioning. In mouse hearts, swiprosin-1 protein expression was increased after 4 and 7 days in myocardial infarct areas specifically in cardiomyocytes as verified by immunoblotting and histology. In rat hearts, swiprosin-1 mRNA expression was induced within 7 days after ischemia/reperfusion but this induction was abrogated by conditioning. As in cultured cardiomyocytes, the expression of swiprosin-1 was associated with a coinduction of arrestin-2, suggesting a common mechanism of regulation. Rno-miR-32-3p and rno-miR-34c-3p were associated with the regulation pattern of both molecules. Moreover, induction of swiprosin-1 and ssc-miR-34c was also confirmed in the infarct zone of pigs. In summary, our data show that up-regulation of swiprosin-1 appears in the postischemic heart during cardiac remodeling and repair in different species.
\end{abstract}

Keywords: cardiac regeneration; cardiac protection; miR-34

\section{Introduction}

Swiprosin-1, also known as EF-Hand domain family member D2 (EFhd2), acts as a calcium sensor that stabilizes F-actin filaments by blocking binding sites of cofilin [1]. Cofilin is needed for the depolymerization of F-actin [2]. Expression of swiprosin-1 has already been identified in 
lymphocytes, B-cells, mast cells, T-cells, neurons, podocytes, and cardiomyocytes [3-9]. Furthermore, a drosophila-specific homologue of human swiprosin-1 participates in skeletal muscle formation [10]. In immune cells, it triggers the formation of lamellopodia, which is required for migration of macrophages [11]. In neurons, swiprosin-1 regulates the cargo capacity of kinesin and participates in neuronal outgrow [1]. In cardiomyocytes, swiprosin-1 is required for the formation of pseudopodia-like structures [9,12]. Pseudopodia-like structures enable adult rat ventricular cardiomyocytes to adapt to culture dishes, to stay alive for days, and to rearrange their sarcomeres by adaptation from the three-dimensional heart to the two-dimensional surrounding of culture dishes. At the level of swiprosin-1 regulation, changes in the mRNA levels went parallel with those of protein expression, suggesting transcriptional regulation. However, the more important consequence of this observation is that this process mimics aspects of cardiac remodeling. Extensive cardiac remodeling with global cardiac changing in gene expression occurs subsequently to myocardial infarction (MI) when repair processes occur [13-15]. Therefore, in the present study we investigated the expression of swiprosin-1 in cardiac tissue during subsequent cardiac remodeling.

Among the common features between cardiac tissue repair and cellular adaptation of terminal differentiated cardiomyocytes to culture conditions are a desensitization of $\beta$-adrenoceptors that can be modified by ischemic postconditioning (IPoC) [16]. Similarly, $\beta$-adrenoceptor responsiveness is reduced during cellular adaptation of cardiomyocytes to cultivation. In line with this observation, swiprosin-1 was found to be strongly coregulated under culture conditions with molecules that regulate $\beta$-adrenoceptor responsiveness, such as $\beta$-arrestin-2 [9]. This coregulation suggests a common mechanism of regulation for both genes. Nowadays, micro-RNAs (miRs) have been identified as potential molecules that coordinate the several proteins acting together to modify the phenotype of individual cells. They bind to mRNA and abrogate subsequent translation into protein. Therefore, we also screened for potential miRs that may commonly be involved in the coregulation of swiprosin-1 and $\beta$-arrestin-2.

\section{Results}

\subsection{Swiprosin-1 Expression In Post-Infarcted Mice}

Although swiprosin-1 expression and its role in cellular adaptation has been documented before [9], the relevance of these in vitro findings for cardiac repair processes remained unclear. Therefore, we first investigated the expression of swiprosin-1 in post-MI hearts. Experiments were performed on mouse hearts previously used for investigating the role of oncostatin $\mathrm{M}$ in cardiac repair, because in cultured cardiomyocytes swiprosin-1 and oncostatin $\mathrm{M}$ are coregulated. Immunoblotting of myocardial tissue samples of infarcted mice specified an increased expression of swiprosin- 1 within the infarcted region when compared with the noninfarcted remote myocardium (Figure 1A). Of note, elevated levels of swiprosin-1 were maintained throughout the first week of cardiac remodeling (Figure 1A). Immunofluorescence analysis of infarcted hearts further allocated increased expression of swiprosin-1 in preserved cardiomyocytes surrounding the infarct scar, whereas swiprosin-1 was barely detectable in cardiomyocytes of distal noninfarcted regions (Figure 1B).

\subsection{Induction of Swiprosin-1 in Rat Hearts after Ischemia/Reperfusion}

In order to address the question of whether induction of cardiac expression of swiprosin- 1 in post ischemic hearts is related to the extent of cardiac repair, swiprosin-1 mRNA expression was analyzed next in rat hearts 7 days after ischemia/reperfusion (I/R). These hearts have previously been used to establish the effect of ischemic pre- and postconditioning on cardiac remodeling [17]. Here, we show that swiprosin-1 mRNA expression is induced by I/R. However, either ischemic preconditioning (IPC) or IPoC abrogate this induction (Figure 2A). IPC and IPoC have proven effects on infarct sizes as quantified by troponin I plasma levels $1 \mathrm{~h}$ after reperfusion [17]. The mRNA expression of swiprosin-1 showed a linear relationship to the infarct size, suggesting that the amount of expression is related 
to cardiac repair processes (Figure 2B). In vitro, swiprosin-1 mRNA expression in cardiomyocytes is associated with a similar expression level of arrestin-2. In the cardiac tissue used to quantify the mRNA expression of swiprosin-1, a similar linear correlation between swiprosin-1 and arrestin-2 was found (Figure 2C).

A

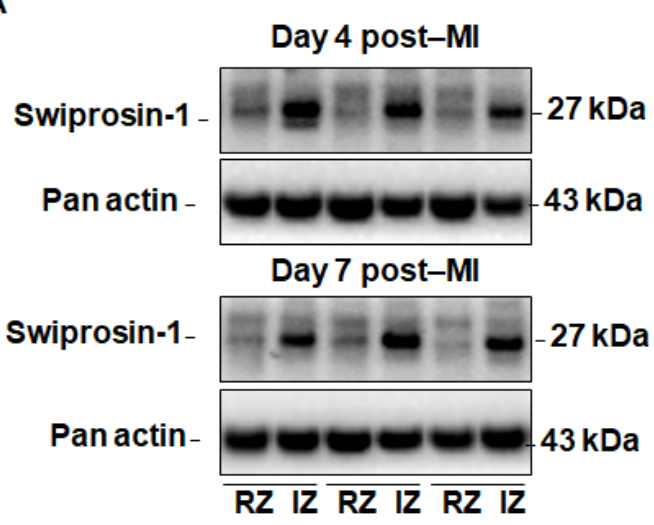

B

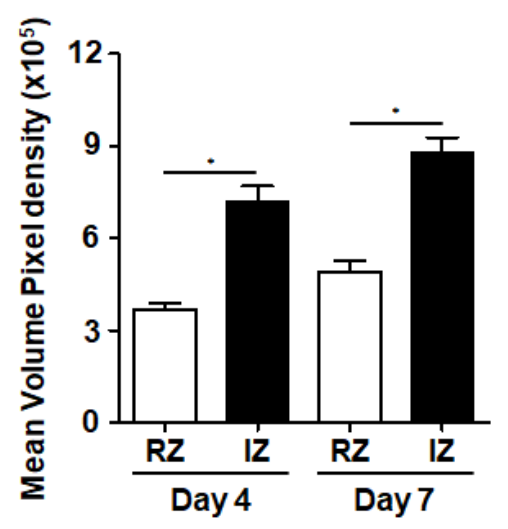

C

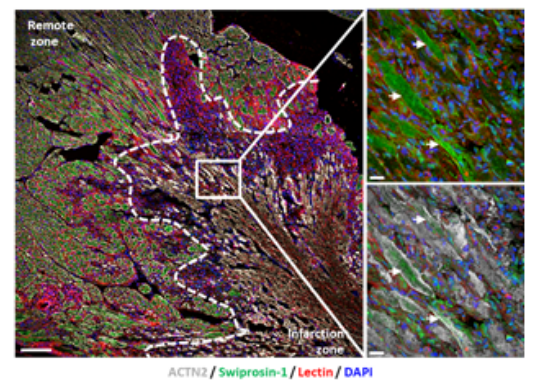

Figure 1. Expression of swiprosin-1 after the onset of myocardial infarction in adult mice. (A) Representative Western Blot analysis of swiprosin-1 in myocardial tissue from adult mice fractionated in noninfarct remote zone (RZ) and infarction zone (IZ) at days 4 and 7 after myocardial infarction (post-MI). (B) Quantification of the two Western Blots showed by A with *, $p<0.05$ vs. RZ; (C) Immunofluorescence analysis of swiprosin-1 (green) in infarcted hearts from mice 4 days post-MI. Viable cardiomyocytes were stained with ACTN2 (grey) and lectin (red). 4',6-diamidino-2-phenylindole (DAPI) was used for nuclear staining (blue). White arrows classify swiprosin-1 immunopositive cardiomyocytes. Scale bars: $100 \mu \mathrm{m}$ and $20 \mu \mathrm{m}$ in magnified sections. 
A

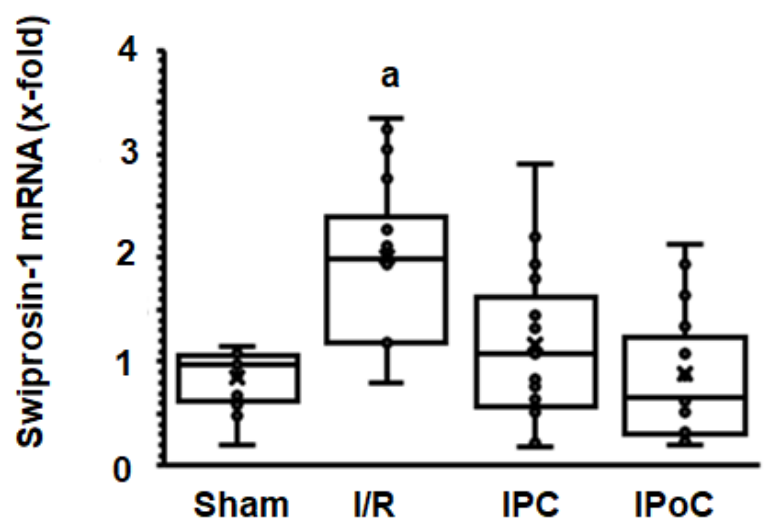

B

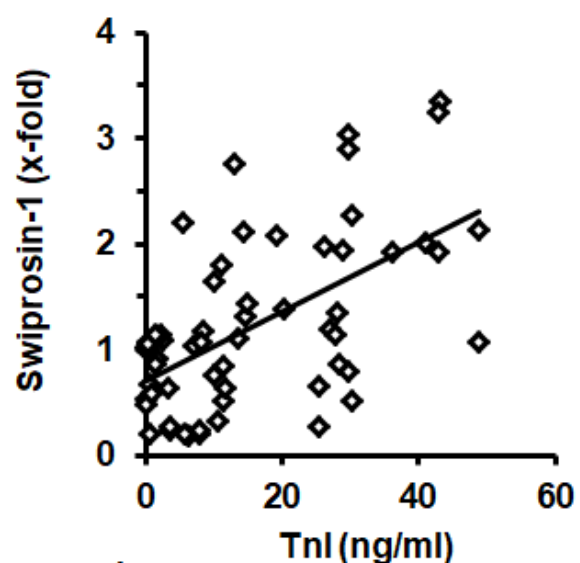

C

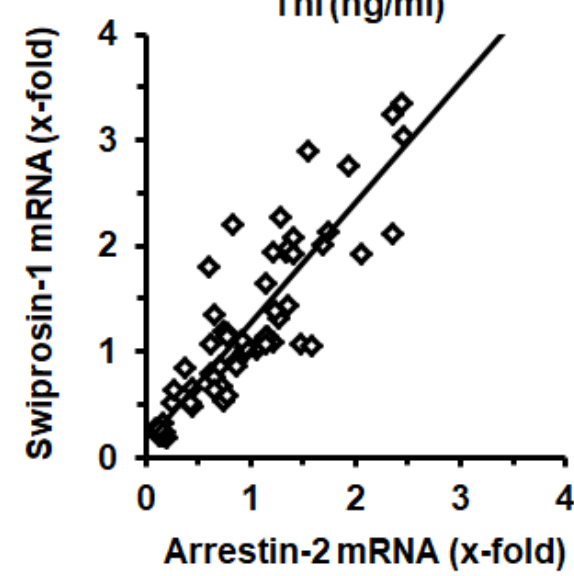

Figure 2. Expression of swiprosin-1 mRNA in the left ventricle from rats seven days after I/R. (A), Box plots showing the level of expression and distribution of samples from sham surgery (Sham), ischemia/reperfusion (I/R), ischemic preconditioning (IPC), and ischemic postconditioning (IPoC). Data are analyzed by One-Way-ANOVA with Student-Newman-Keuls post-hoc analysis ( $\mathrm{a} ; p<0.05$ vs. all other groups). (B); Correlation between infarct sizes as quantified by troponin I release into the plasma $60 \mathrm{~min}$ after reperfusion and swiprosin-1 mRNA expression. (C) Correlation between arrestin-2 mRNA expression and swiprosin-1 mRNA expression.

Coregulation of swiprosin- 1 and arrestin-2 suggests a common mechanism of regulation, such as by miR. In the rat heart tissue used for this study, 364 miRs were constitutively detected in all samples. Among them, 27 miRs were either positively $(n=15)$ or negatively $(n=12)$ associated with the mRNA expression of swiprosin-1 (Table 1). However, only 12 of 27 miRs showed an ischemia/reperfusion-dependent regulation such as swiprosin-1, and only in two cases could the induction of miR be abrogated by IPC or IPoC as found for swiprosin-1. These two most likely candidates were rno-miR-32-3p and rno-miR-34c-3p (Figure 3A,B). 
Table 1. Correlation between swiprosin-1 mRNA and micro-RNAs (miRs).

\begin{tabular}{|c|c|c|c|c|}
\hline rno-miR & R-Coefficient & $p$-Value & $\mathbf{I} / \mathbf{R}$ & IPC/IPoC \\
\hline \multicolumn{5}{|c|}{ Positive Correlation } \\
\hline $32-3 p$ & $r=0.596$ & $p=0.003$ & yes & yes \\
\hline $34 c-3 p$ & $r=0.495$ & $p=0.016$ & yes & yes \\
\hline $18 a-3 p$ & $r=0.465$ & $p=0.025$ & yes & no \\
\hline $23 b-5 p$ & $r=0.475$ & $p=0.022$ & yes & no \\
\hline $27 b-5 p$ & $r=0.436$ & $p=0.038$ & yes & no \\
\hline $100-3 p$ & $r=0.431$ & $p=0.040$ & no & - \\
\hline $146 a-5 p$ & $r=0.454$ & $p=0.029$ & no & - \\
\hline $296-5 p$ & $r=0.474$ & $p=0.022$ & no & - \\
\hline $324-5 p$ & $r=0.520$ & $p=0.011$ & no & - \\
\hline $342-3 p$ & $r=0.516$ & $p=0.012$ & no & - \\
\hline $455-3 p$ & $r=0.453$ & $p=0.030$ & no & - \\
\hline $497-5 p$ & $r=0.458$ & $p=0.028$ & no & - \\
\hline $505-5 p$ & $r=0.436$ & $p=0.038$ & no & - \\
\hline $532-3 p$ & $r=0.514$ & $p=0.012$ & no & - \\
\hline $674-5 p$ & $r=0.616$ & $p=0.002$ & no & - \\
\hline \multicolumn{5}{|c|}{ Negative Correlation } \\
\hline \multicolumn{5}{|l|}{$7 \mathrm{~b}$} \\
\hline $24-2-5 p$ & $r=-0.475$ & $p=0.022$ & no & - \\
\hline $29 b-3 p$ & $r=-0.425$ & $p=0.043$ & yes & no \\
\hline $33-5 p$ & $r=-0.563$ & $p=0.005$ & yes & no \\
\hline $99 a-5 p$ & $r=-0.431$ & $p=0.040$ & yes & no \\
\hline $145-3 p$ & $r=-0.520$ & $p=0.011$ & yes & no \\
\hline $150-5 p$ & $r=-0.414$ & $p=0.050$ & yes & no \\
\hline $186-5 p$ & $r=-0.425$ & $p=0.043$ & no & - \\
\hline $194-5 p$ & $r=-0.495$ & $p=0.016$ & yes & no \\
\hline $339-3 p$ & $r=-0.447$ & $p=0.033$ & yes & no \\
\hline $345-3 p$ & $r=-0.426$ & $p=0.042$ & no & - \\
\hline $3068-5 p$ & $r=-0.422$ & $p=0.045$ & yes & no \\
\hline
\end{tabular}

As expected from the observed coregulation, the mRNA expression of swiprosin-1 showed a linear correlation with the amount of rno-miR-32-3p and rno-miR-34c-3p in these samples (Figure 4A). Moreover, both miRs showed a linear correlation with the infarct size (quantified as troponin I plasma concentration $1 \mathrm{~h}$ after $\mathrm{I} / \mathrm{R}$ ) and with arrestin-2 mRNA expression as well (Figure 4B,C).

In the cardiac tissue used for this analysis, mRNA expression of 72 different genes related to cardiac biology was analyzed (see ref. 17). A detailed analysis of further coregulated mRNAs with both miRs was performed by investigating a potential linear correlation between mRNAs and miRs. Interestingly, rno-miR-32-3p showed a positive correlation with genes associated with hypertrophy and fibrosis and a negative correlation with sodium-calcium exchanger (NCX) (Table 2). The data suggest that the increased expression of rno-miR-32-3p contributes to the maladaptive phenotype of cardiac adaptation in these hearts. In contrast, rno-miR-34c-3p displayed a nearly exclusive coregulation with swiprosin-1 and arrestin-2 (Table 2). 
A

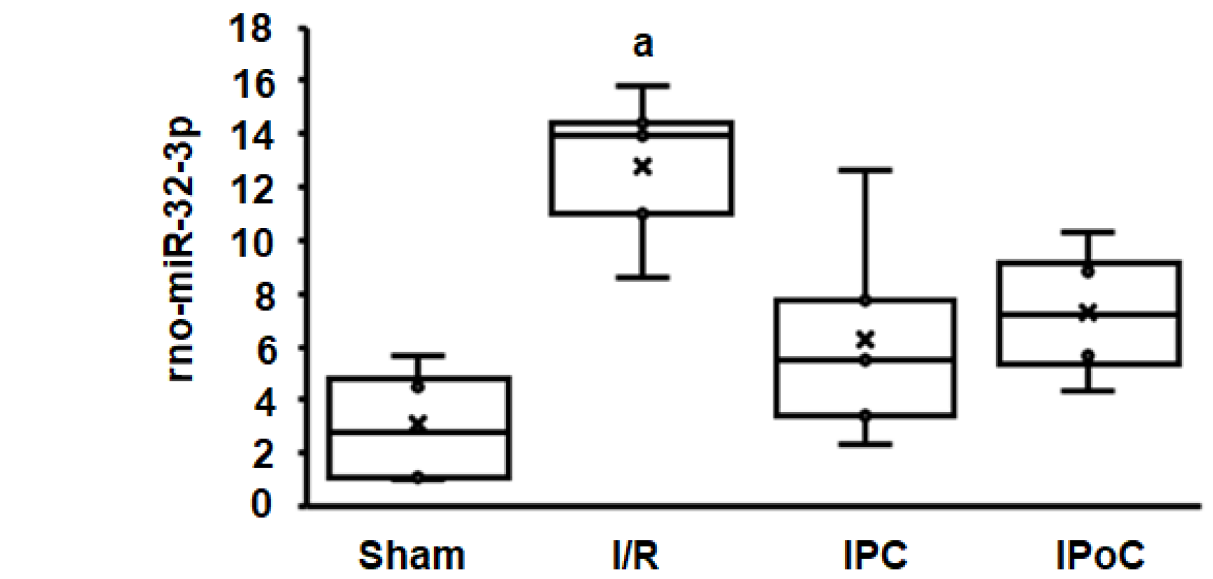

B

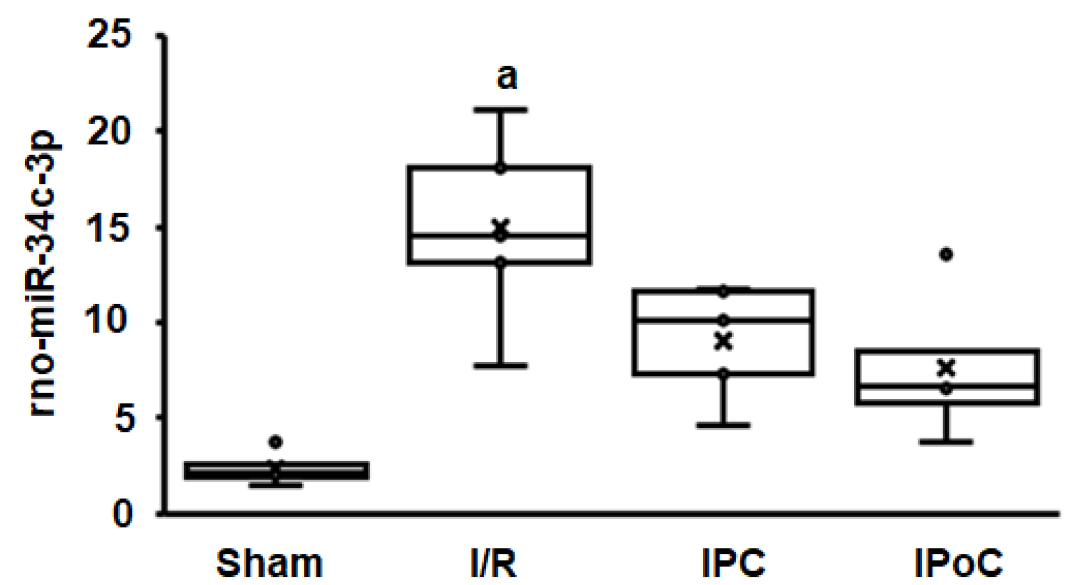

Figure 3. Expression of rno-miR-32-3p (A) and rno-miR-34c-3p (B) in the left ventricle from rats seven days after I/R. A). Box plots showing the level of expression and distribution of samples from sham surgery (Sham), ischemia/reperfusion (I/R), ischemic preconditioning (IPC), and ischemic postconditioning (IPoC). Data are analyzed by One-Way-ANOVA with Student-Newman-Keuls posthoc analysis (a; $p<0.05$ vs. all other groups).

A

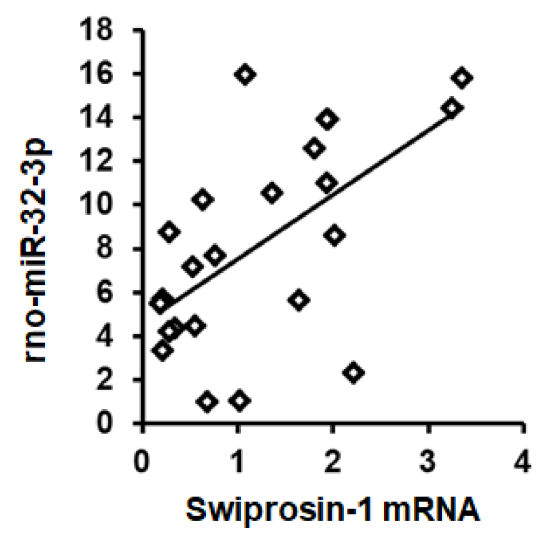

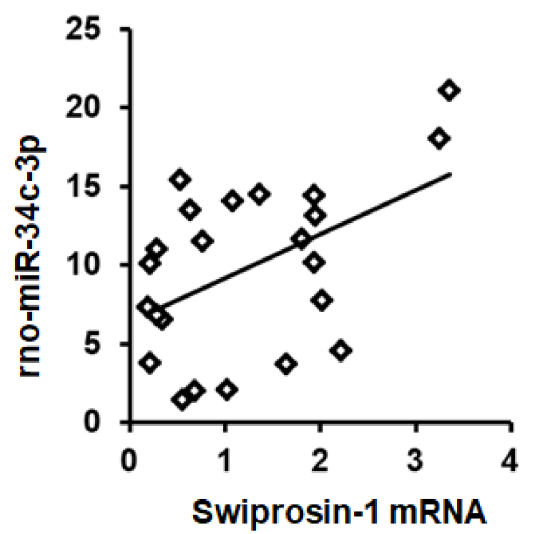

Swiprosin-1 mRNA

Figure 4. Cont. 
B

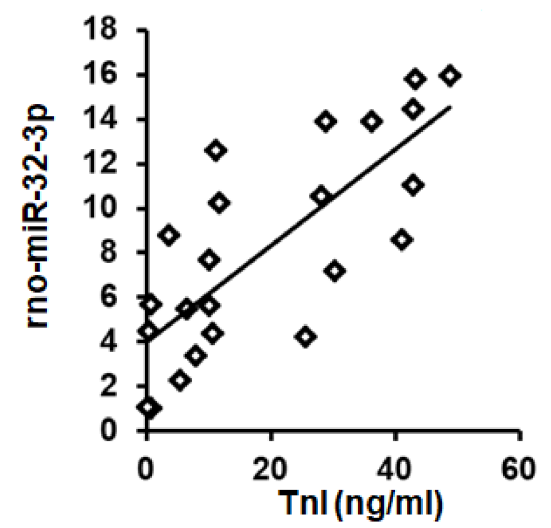

c

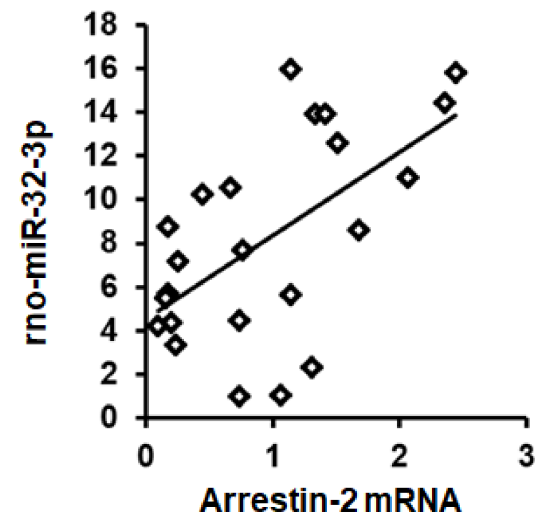

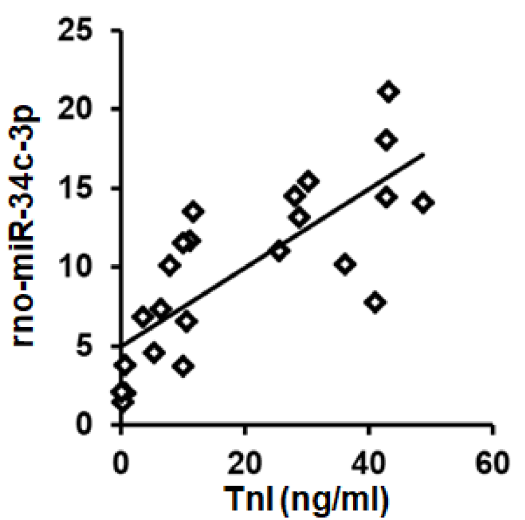

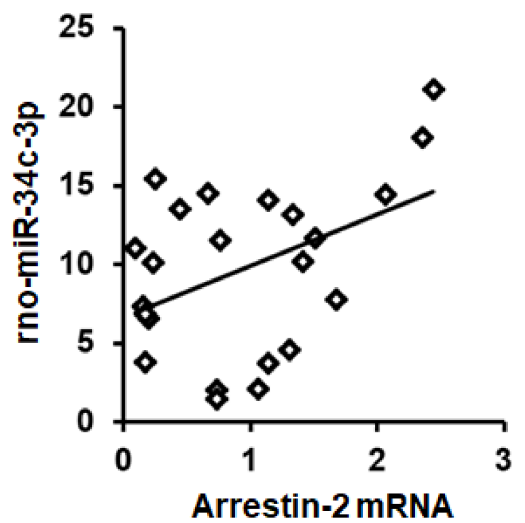

Figure 4. Correlation between swiprosin-1 mRNA (A), infarct size (as quantified by troponin I plasma concentration) (B), and arrestin-2 (C), with the abundancy of rno-miR-32-3p and rno-miR-34c-3p.

Table 2. Correlation between miRs and mRNAs.

\begin{tabular}{ccc}
\hline \multicolumn{3}{c}{ rno-miR-32-3p } \\
\hline Swiprosin-1 & $r=0.596$ & $p=0.003$ \\
\hline Arrestin-2 & $r=0.585$ & $p=0.003$ \\
\hline Biglycan & & \\
\hline Collagen-1 & $r=0.581$ & $p=0.004$ \\
\hline Collagen-3 & $r=0.515$ & $p=0.020$ \\
\hline Fibronectin & $r=0.452$ & $p=0.030$ \\
\hline Arginase-2 & $r=0.434$ & $p=0.038$ \\
\hline iNOS & & \\
\hline RAMP-3 & & \\
\hline MHC- $\alpha$ & & \\
\hline NCX & & \\
\hline Troponin T & $r=-0.470$ & $p=0.023$ \\
\hline HADHA & & \\
\hline UCP-2 & & \\
\hline Slc5a3 & & \\
\hline \multicolumn{2}{c}{ rno-miR-34c-3p } \\
\hline Swiprosin-1 & $r=0.495$ & $p=0.016$ \\
\hline Arrestin-2 & $r=0.440$ & $p=0.036$ \\
\hline
\end{tabular}


Table 2. Cont.

\begin{tabular}{c}
\hline \multicolumn{1}{c}{ rno-miR-34c-3p } \\
\hline ANP \\
\hline MHC- $\alpha$ \\
\hline Elfn2 \\
\hline Kcnh5 \\
\hline Slc5a3 \\
\hline Socs7
\end{tabular}

Abbreviations: NOS, nitric oxide synthase; RAMP, receptor associated modifying protein; ANP, atrial natriuretic peptide; MHC; myosin heavy chain; Elfn, extracellular leucine rich repeat and fibronectin type III domain containing; Kcnh, potassium voltage-gated channel, subfamily H; Slc, solute carrier; Socs, suppressor of cytokine signaling.

\subsection{Regulation of Swiprosin-1 Expression in Pig Hearts}

The aforementioned experiments have shown that swiprosin- 1 is up-regulated during cardiac repair processes in small rodents. To investigate the relevance for other species as well, the investigation was extended by analysis of swiprosin-1 expression in pig hearts. This analysis was performed on pig heart samples previously analyzed in more detail $[18,19]$. Tissue samples were taken $3 \mathrm{~h}$ and 3 days after reperfusion from the border zone, infarct zone, and unaffected control zone. Again, swiprosin-1 mRNA expression was induced at the late time-point (day 3) in the infarct zone (Figure 5A). In comparison, expression of proinflammatory markers such as MCP-1 was induced already after $3 \mathrm{~h}$ (Figure 5B). Finally, in the reperfused heart, ssc-miR-34c was again increased by ischemia/reperfusion, and this was again abrogated by IPC and IPoC.

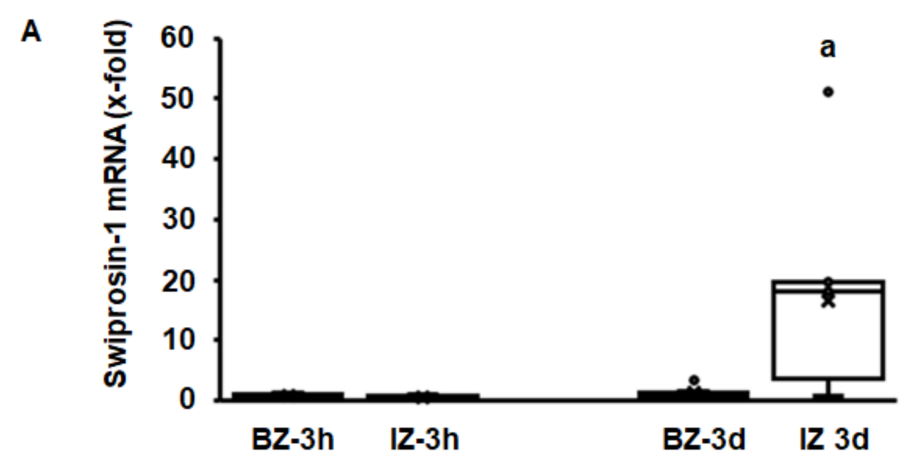

B

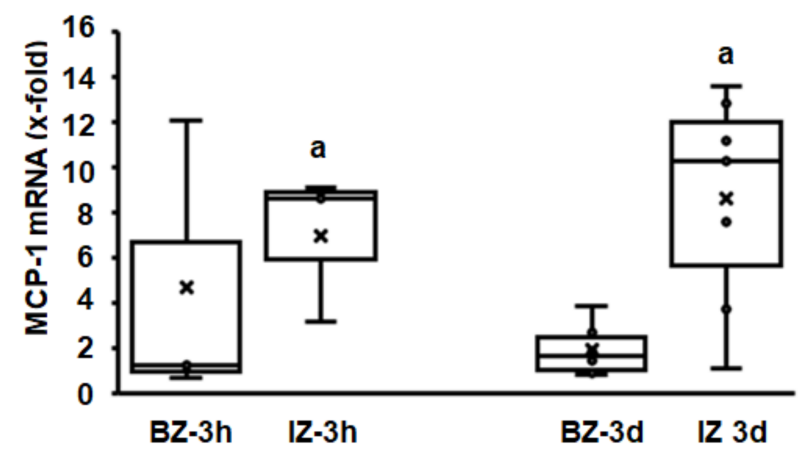

Figure 5. Cont. 
C

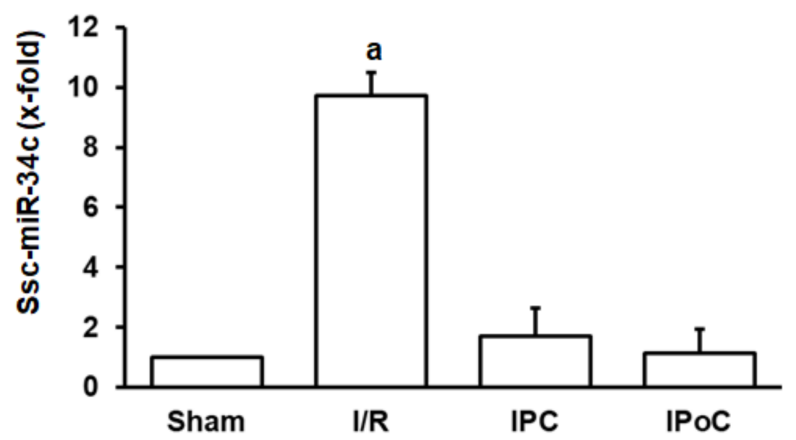

Figure 5. Expression of swiprosin-1, MCP-1, and ssc-miR-34c in the border zone (BZ) or infarct zone (IZ) three hours or 3 days after reperfusion. The quantification for ssc-miR-34c was performed in the infarct zone $3 \mathrm{~h}$ after reperfusion.

\section{Discussion}

Swiprosin-1 is expressed in various tissues, but although its expression and function in adult rat ventricular cardiomyocytes have been reported before, a proof of concept is still lacking that will show that swiprosin- 1 is expressed in cardiac tissue and furthermore differentially regulated during active repair processes. Postinfarct remodeling requires extensive cardiac reconstruction and is therefore suitable to address this question. Using different conditioning protocols (IPC and IPoC), infarct sizes and thereby the amount of repair process can be modified. In line with these assumptions, the main findings of the current study are that swiprosin-1 expression is induced during cardiac repair processes in mice, rats, and pigs. Moreover, the expression of swiprosin- 1 is strongest in those areas of the heart where cardiac remodeling occurs, that is, in the border zone of MI mice hearts and in the infarct zone of reperfused rat and pig hearts. Finally, histology identified cardiomyocytes as the main side of expression.

Our study is based on the assumption that swiprosin expression in cardiomyocytes is transcriptionally regulated. This argument comes from our previous study showing that targeting swiprosin mRNA by siRNA reduces swiprosin protein expression as well. In our current study, we show swiprosin protein expression in mice and confirmed its expression on mRNA in rat and pig. This expression is associated with miR expression, indicating a complex regulatory network in cardiomycytes. Nevertheless, on the basis of the material used in this study we could not directly confirm a strict coregulation of swiprosin mRNA and swiprosin protein for the three species, and this is of course a limitation of the study.

In the process of cardiac remodeling, a coordinative regulation of genes that encode for proteins required for cardiac contractility and excitation is required. As a potential mechanism to coordinate such expression, miRs are interesting molecules as the predicted consensus sequence of these molecules potentially targets multiple mRNAs. It was already known that in cardiomyocytes swiprosin-1 is coregulated with the mRNA expression of proteins linked to $\beta$-adrenoceptor desensitization. A desensitization of $\beta$-adrenoceptors can occur under conditions of cardiac cell cultivation and in the postischemic heart [16]. Nevertheless, coregulation of cardiac expression of swiprosin-1 and arrestin-2 in vivo has not been demonstrated before. Screening potential miRs that may trigger this coregulation, two miRs were identified. Both showed a similar type of regulation of expression to swiprosin-1, namely, rno-miR-32-3p and rno-miR-34c-3p. The amount of expression of both miRs correlates with swiprosin- 1 and arrestin-2.

Rno-miR-32-3p was also associated with other genes that are well known to affect cardiac remodeling. The level of miR expression correlated with genes involved in fibrosis (biglycan, collagen-1, collagen-3, and fibronectin), inflammation (iNOS, arginase-2), and receptor activity (RAMP-3). Biglycan and fibronectin can act as a modulator of TGF- $\beta 1$ activity, and in line with this function the expression of collagen increased $[20,21]$. RAMPs, but not RAMP-3, are differentially expressed in cardiomyocytes under hypertensive conditions [22]. On the other hand, increased expression of rno-miR-32-3p was 
inversely correlated with the expression of sarcomeric proteins (MHC- $\alpha$, troponin $\mathrm{T}$ ), transporters of sodium (NCX, Scl5a3), and that of fatty acid metabolism (HADHA, UCP-2). While HADHA is required for fatty acid metabolism, down-regulation of UCP-2 will favor glucose utilization [23]. Therefore, the down-regulation of HADHA and UCP-2 both initiate a metabolic switch into the direction of glucose metabolism. In general, the common regulation of swiprosin- 1 with other proteins known to be differentially regulated during cardiac remodeling suggests that swiprosin- 1 is part of this program. However, as most of the adaptations (fibrosis, metabolic switch, and sarcomere remodeling) are associated with maladaptive function, the overall function of swiprosin-1 may be maladaptive as well. As swiprosin-1 participates in cardiomyocyte dedifferentiation, a mechanism that leads to impaired cardiac function when occurring in a chronic rather than acute way, this may indeed be the case. In this way, the lower expression of swiprosin-1 after IPC and IPoC will be the consequence of smaller infarct sizes.

In contrast, rno-miR-34c-3p was not associated with maladaptive gene reprogramming such as rno-miR-32p. This miR, in addition to swiprosin-1, was associated with down-regulation of four different ion transporters. Of note, $\mathrm{I} / \mathrm{R}$-dependent regulation of miR-34c was found in rat and pig tissues. Finally, both miRs identified in this study have not yet been associated with myocardial remodeling and represent another new finding of this study.

The design of the current study does not allow identifying potential mechanisms by which swiprosin-1 may affect the remodeling process. However, it has been shown before that dedifferentiation processes are needed for regeneration of cardiomyocytes. Such processes depend on oncostatin M as well as of swiprosin-1 [24]. In this context, it was also shown that swiprosin- 1 and oncostatin $M$ are coexpressed and coregulated in cardiomyocytes in vitro [12]. Oncostatin $\mathrm{M}$ may link the initial inflammatory process during cardiac repair to cardiac remodeling, and this process may require swiprosin-1 to arrange sarcomeric structures.

In the current study, swiprosin expression was found in the same region of mouse tissue and at the same time-point as oncostatin M (this study and ref. 24). Oncostatin M is released by immune cells like macrophages and triggers the release of Reg $3 \beta$ from cardiomyocytes. Reg $3 \beta$ directs more oncostatin $M$, releasing immune cells to this area. At the same time, oncostatin $M$ triggers the dedifferentiation of cardiomyocytes. This process requires swiprosin [9]. Therefore, oncostatin M-producing immune cells like macrophages may potentiate the induction of swiprosin expression at the site of cardiac differentiation.

In conclusion, this study highlights the tissue-specific expression of swiprosin- 1 in postinfarct hearts, and the way of regulation suggests an important role in cardiac repair.

\section{Materials and Methods}

\subsection{Animal Experiments}

All types of analysis in this study were performed on tissue samples from previous experiments. Therefore, no new animal experiments were performed for this study. The samples for analysis of mice tissue were generated in a mouse MI model as described before [24]. Briefly, the model used a permanent ligature of the LAD in mice with a subsequent 4 and 7 days follow-up before the mice were sacrificed. All relevant functional data were published before [24]. The samples for analysis of rat tissue were generated in a rat model of ischemia/reperfusion as described [16]. This model uses a 30 min LAD occlusion with subsequent reperfusion for 7 days. All relevant functional data were published before $[16,17]$. Infarct sizes were quantified by increased plasma troponin I levels $60 \mathrm{~min}$ after reperfusion as described before [16]. The samples for analysis of pig tissue were generated in a pig model of $\mathrm{I} / \mathrm{R}$ as described $[18,19]$. This model is based on a $90 \mathrm{~min}$ percutaneous balloon occlusion of the left anterior descending coronary artery, followed by balloon deflation in anaesthetized pigs and subsequent reperfusion for $3 \mathrm{~h}$ or $3 \mathrm{~d}$. 


\subsection{Western Blot and Histology on Mouse Samples}

Infarcted hearts of adult mice were harvested and dissected in an infarction zone and remote myocardium (basal part of the interventricular septum) for Western Blot analysis. Fractionated samples were sonicated in lysis buffer (0.1 M Tris-HCl pH 8.8, $0.01 \mathrm{M}$ EDTA, $0.04 \mathrm{M}$ DTT, $10 \%$ SDS, and pH 8.0) supplemented with protease inhibitors. Twenty micrograms of each protein sample were loaded on Gradient NuPAGE 4-12\% Bis-Tris gels (Invitrogen) to perform SDS-PAGE. After transferring onto nitrocellulose membranes (Invitrogen), immunoreactive proteins were visualized on the ChemiDocXRS+ System (BioRad) and analyzed by using the Image Lab software (BioRad). The following antibodies for Western Blot analysis were used: goat anti-Swiprosin-1 antibody (Sigma-Aldrich, catalogue no. SAB2501007) and rabbit anti-Pan-actin antibody (Cell Signaling Technology, catalogue no. 4968). Secondary antibodies conjugated with horseradish peroxidase were purchased from R\&D Systems.

The method for immunohistochemical analysis of murine infarcted hearts has been previously described (24). The following antibodies for immunofluorescence staining were used: goat anti-Swiprosin-1 antibody (Sigma-Aldrich, catalogue no. SAB2501007) and rabbit anti- $\alpha$ -sarcomeric-actinin monoclonal antibody (Clone: EA-52, Sigma-Aldrich, catalogue no. A7732). For nuclear counterstain, 4', 6 -diamidino-2-phenylindole (DAPI) was used (Sigma Aldrich, catalogue no. D9542). Lectin from Ulex europaeus (L4895; catalogue no. L4895; Sigma Aldrich) was used to detect intact cells.

\subsection{RT-PCR on Rat and Pig Tissue}

The method on RNA isolation and real-time reverse-transcriptase polymerase chain reaction (RT-PCR) used to quantify the mRNA expression has been described before in detail [16]. A list of all rat primers used in the study has also published before [17] Table 3.

Table 3. List of porcine primers used in this study.

\begin{tabular}{ccc}
\hline Beta-2-Mircoglobulin & forward: CGT GGC CTT GGT CCT GCT CG & reverse: TCC GTT TTC CGC TGG TGT GC \\
\hline EFhD2 & forward: TCC GGG AGT TCC TCC TGA TT & reverse: AAG CTC TTC GCT CCC TTG AC \\
\hline MCP-1 & forward: CAG CCA CCT TVT GCA CCC AGG & Reverse: CAC AGA TCT CCT TGCCCG CGA \\
\hline
\end{tabular}

\subsection{Statistics}

Group comparison was performed by One-Way-ANOVA and Student-Newman-Keuls post hoc analysis. If the probability to belong to equal groups is below $p \leq 0.05$, this is indicated by different letters in the figures. Correlation analysis was performed by Pearson correlation. Exact $p$ values for correlation analysis are given in the figures to the legend.

Author Contributions: Data curation, Z.G., A.M., R.S. (Rolf Schreckenberg), J.P., H.L., K.K. and K.-D.S.; Formal analysis, A.M., R.S. (Rolf Schreckenberg), P.B. and K.-D.S.; Funding acquisition, T.B., R.S. (Rainer Schulz) and P.F.; Investigation, J.P. and H.L.; Methodology, Z.G., A.M., R.S. (Rolf Schreckenberg), J.P. and P.B.; Project administration, R.S. (Rolf Schreckenberg), P.B., T.B. and R.S. (Rainer Schulz); Software, Z.G. and K.K.; Supervision, K.-D.S.; Validation, R.S. (Rolf Schreckenberg) and K.K.; Writing—original draft, K.-D.S.; Writing—review \& editing, R.S. (Rainer Schulz) and P.F. All authors have read and agreed to the published version of the manuscript.

Funding: KDS: RS and TB are supported by the Sonderforschungsbereich 1213 of the German Research Foundation (DFG). This work was supported by the National Research, Development, and Innovation Office of Hungary (MYOTeam GINOP-2.3.2-15-2016-00040 (to PB); National Heart Program NVKP 16-1-2016-0017 (to PF) and by the Higher Education Institutional Excellence Program of the Ministry of Human Capacities in Hungary, within the framework of the Therapeutic Development thematic program of the Semmelweis University, Budapest, Hungary (to PF). ZG is supported by a János Bolyai Research Scholarships of the Hungarian Academy of Sciences and by the ÚNKP-19-4 New National Excellence Program of the Ministry of Human Capacities. MA was supported by the New National Excellence Program of the Ministry of Human Capacities (ÚNKP-19-3-I-SE-60).

Acknowledgments: The authors thank Nadine Woitasky and Karin Westermann for excellent technical support. Conflicts of Interest: P.F. is the founder and CEO and PB is employed by Pharmahungary Group, a group of R\&D companies. 


\section{Abbreviations}

IPC Ischemic Preconditioning

IPoC Ischemic Postconditioning

I/R Ischemia/Reperfusion

LAD Left anterior descending coronary artery

MI Myocardial Infarction

\section{References}

1. Mielenz, D.; Gunn-Moore, F. Physiological and pathophysiological functions of Swiprosin-1/ EFhd2 in the nervous system. Biochem. J. 2016, 476, 2429-2437. [CrossRef] [PubMed]

2. Huh, Y.H.; Kim, S.H.; Chung, K.H.; Oh, S.; Kwon, M.S.; Choi, H.W.; Rhee, S.; Ryu, J.H.; Park, Z.Y.; Jun, C.D.; et al. Swiprosin-1 modulates actin dynamics by regulating the F-actin accessibility to cofilin. Cell. Mol. Life Sci. 2013, 70, 4841-4854. [CrossRef] [PubMed]

3. Vuadens, F.; Rufer, N.; Kress, A.; Corthesy, P.; Schneider, P.; Tissot, J.D. Identification of swiprosin 1 in human lymphocytes. Proteomics 2014, 4, 2216-2220. [CrossRef] [PubMed]

4. Mielenz, D.; Vettermann, C.; Hampel, M.; Lang, C.; Avramidou, A.; Karas, M.; Jäck, H.M. Lipid rafts associate with intracellular B cell receptors and exhibit a B cell stage-specific protein composition. J. Immunol. 2005, 175, 3508-3517. [CrossRef]

5. Thylur, R.P.; Kim, Y.D.; Kwon, M.S.; Oh, H.M.; Kwon, H.K.; Kim, S.H.; Im, S.H.; Chun, J.S.; Park, Z.Y.; Jun, C.D. Swiprosin-a is expressed in mast cells and up-regulated through the protein kinase C beta I/eta pathway. J. Cell. Biochem. 2009, 108, 705-715. [CrossRef]

6. Kim, Y.D.; Kwon, M.S.; Na, B.R.; Kim, H.R.; Lee, H.S.; Jun, C.D. Swiprosin-1 expression is up-regulated through protein kinase C- $\theta$ and NF-KB pathway in T cells. Immune Netw. 2013, 13, 55-62. [CrossRef]

7. Purohit, P.; Perez-Branguli, F.; Prots, I.; Borger, E.; Gunn-Moore, F.; Welzel, O.; Loy, K.; Wenzel, E.M.; Grömer, T.W.; Brachs, S.; et al. The $\mathrm{Ca}^{2+}$ Sensor Protein Swiprosin-1/EFhd2 Is Present in Neurites and Involved in Kinesin-Mediated Transport in Neurons. PLoS ONE 2014, 18, e103976. [CrossRef]

8. Wang, R.M.; Wang, Z.B.; Wang, Y.; Liu, W.Y.; Li, Y.; Tong, L.C.; Zhang, S.; Su, D.F.; Cao, Y.B.; Li, L.; et al. Swiprosin-1 promotes mitochondrial-dependent apoptosis of glomerular podocytes via p38 MAPK pathway in early-stage diabetic nephropathy. Cell. Physiol. Biochem. 2018, 45, 899-916. [CrossRef]

9. Nippert, F.; Schreckenberg, R.; Hess, A.; Weber, M.; Schlüter, K.-D. The effects of swiprosin-1 on the formation pf pseudopodia-like structures and $\beta$-adrenoceptor coupling in cultured adult rat ventricular cardiomyocytes. PLoS ONE 2016, 11, e0167655. [CrossRef]

10. Hombruch-Freitag, C.; Griemert, B.; Buttgereit, D.; Renkawitz-Pohl, R. Drosophila swiprosin-1/EFHD2 accumulates at the perfusion complex stage during Drosophila myoblast fusion. J. Cell Sci. 2011, 124, 3266-3278. [CrossRef]

11. Tu, Y.; Zhang, L.; Tong, L.; Wang, Y.; Zhang, S.; Wang, R.; Li, L.; Wang, Z. EFhd2/swiprosin-1 regulates LPS-induced macrophage recruitment via enhancing actin polymerization and cell migration. Int. Immunopharmacol. 2018, 55, 263-271. [CrossRef] [PubMed]

12. Nippert, F.; Schreckenberg, R.; Schlüter, K.-D. Isolation and cultivation of adult rat cardiomyocytes. J. Vis. Exp. 2017, 128, e56634. [CrossRef] [PubMed]

13. Perrino, C.; Barabasi, A.L.; Condorelli, G.; Davidson, S.M.; de Windt, L.; Dimmeler, S.; Engel, F.B.; Hausenloy, D.J.; Hill, J.A.; van Laake, L.W.; et al. Epigenomic and transcriptomic approaches in the post-genomic era: Path to novel targest for diagnosis and therapy of the ischaemic heart? Position paper of the European society of cardiology working group on cellular biology of the heart. Cardiovasc. Res. 2017, 113, 723-736. [CrossRef] [PubMed]

14. Shamagian, G.; Madonna, R.; Taylor, D.; Climent, A.M.; Prosper, F.; Bras-Rosario, L.; Bayes-Genis, A.; Ferdinandy, P.; Fernandez-Aviles, F.; Izpisua Belmonte, J.C.; et al. Perspectives on directions and priorities for future studies in regenerative medicine. Circ. Res. 2019, 124, 938-951. [CrossRef]

15. Madonna, R.; van Laake, L.W.; Davidson, S.M.; Engel, F.B.; Hausenloy, D.J.; Lecour, S.; Leor, J.; Perrino, C.; Schulz, R.; Ytrehus, K.; et al. Position paper of the European society of cardiology working group cellular biology on the heart: Cell-based therapies for myocardial repair and regeneration in ischemic heart disease and heart failure. Eur. Heart J. 2016, 37, 1789-1798. [CrossRef] 
16. Schreckenberg, R.; Bencsik, P.; Weber, M.; Abdallah, Y.; Csonka, C.; Gömöri, K.; Kiss, K.; Paloczi, J.; Pipis, J.; Sarközy, M.; et al. Adverse effects on $\beta$-adrenergic receptor coupling: Ischemic postconditioning failed to preserve long-term cardiac function. J. Am. Heart Assoc. 2017, 6, e006809. [CrossRef]

17. Schreckenberg, R.; Klein, J.; Kutsche, H.S.; Schulz, R.; Gömöri, K.; Bencsik, P.; Bencsik, B.; Agg, B.; Saghy, E.; Ferdinandy, P.; et al. Ischemic post-conditioning in rats: Responder and nonresponder differ in transcriptome of mitochondrial proteins. J. Cell. Mol. Med. 2020, in press. [CrossRef]

18. Baranyai, T.; Giricz, Z.; Varga, Z.V.; Koncsos, G.; Lukovic, D.; Makkos, A.; Sarközy, M.; Pavo, N.; Jakab, A.; Czimbalmos, C.; et al. In vivo MRI and ex vivo histology assessment of the cardioprotection induced by ischemic preconditioning, postconditioning and remote conditioning in a closed-chest procine model of reperfused acute myocardial infarction: Importance of microvasculature. J. Transl. Med. 2017, 15, 67. [CrossRef]

19. Lukovic, D.; Gugerell, A.; Zlabinger, K.; Winkler, J.; Pavo, N.; Baranyai, T.; Giricz, Z.; Varga, Z.V.; Riesenhuber, M.; Spannbauer, A.; et al. Transcriptional Alterations by Ischaemic Postconditioning in a Pig Infarction Model: Impact on Microvascular Protection. Int. J. Mol. Sci. 2019, 20, 344. [CrossRef]

20. Westermann, D.; Mersmann, J.; Melchior, A.; Freudenberger, T.; Patrik, C.; Schaefer, L.; Lüllmann-Rauch, R.; Letaau, O.; Jacoiby, C.; Schrader, J.; et al. Biglycan is required for adaptive remodeling after myocardial infarction. Circulation 2008, 117, 1269-1276. [CrossRef]

21. Frangogiannis, N. The extracellular matrix in ischemic and nonischemic heart failure. Circ. Res. 2019, 125, 117-146. [CrossRef]

22. Schlier, A.; Schreckenberg, R.; Abdallah, Y.; Krasteva, G.; Piper, H.M.; Pfeil, U.; Kummer, W.; Schlüter, K.-D. CGRP- $\alpha$ responsiveness of adult rat ventricular cardiomyocytes from normotensive and spontaneously hypertensive rats. Eur. J. Cell Biol. 2009, 88, 227-241. [CrossRef] [PubMed]

23. Kutsche, H.S.; Schreckenberg, R.; Weber, M.; Hirschhäuser, C.; Rohrbach, S.; Li, L.; Niemann, B.; Schulz, R.; Schlüter, K.-D. Alteration in glucose metabolism during the transition to heart failure: The contribution of UCP-2. Cells 2020, 9, 552. [CrossRef] [PubMed]

24. Lörchner, H.; Pöling, J.; Gajawada, P.; Hou, Y.; Polyakova, V.; Kostin, S.; Adrian-Segarra, J.M.; Boettger, T.; Wietelmann, A.; Warnecke, H.; et al. Myocardial healing requires Reg3 $\beta$-dependent accumulation of macrophages in the ischemic heart. Nat. Med. 2015, 21, 353-362. [CrossRef] [PubMed] 